\title{
A Rare Case of Posterior Interosseous Nerve Palsy Post-venepuncture
}

\author{
Raymond D.K. Yeak ${ }^{1}$, Yee Yee Yap ${ }^{2}$ and Nizlan M. Nasir ${ }^{1}$ \\ ${ }^{1}$ Department of Orthopaedic Surgery, Universiti Putra Malaysia, Serdang, Selangor, Malaysia \\ ${ }^{2}$ Department of Haematology, Ampang Hospital, Ampang Jaya, Selangor, Malaysia
}

\begin{abstract}
Wrist drop post-venepuncture is uncommon. There has been reported cases of direct injury to the radial nerve during venepuncture but to our knowledge, there has never been a case of posterior interosseous nerve (PIN) injury. A 34-year female, right hand dominant homemaker, with a newly diagnosed diabetes mellitus, was admitted for diabetic ketoacidosis. There was difficult access with multiple attempts in her blood taking over the antebrachial fossa and forearm. Thereafter, she was unable to fully extend her wrist, fingers and thumb with an intact sensation. The electrophysiological study was suggestive of demyelinating right radial neuropathy at the elbow. Despite the transient blood taking session, patient developed neuropraxia, which only resolved after four months. We wish to report this case of unusual presentation of a PIN palsy post- routine venepuncture, which can result in high morbidity to a patient.
\end{abstract}

Key Words: Posterior interosseous nerve syndrome, venepuncture, neuropraxia, wrist drop.

How to cite this article: Yeak RD, Yap YY, Nasir NM. A Rare Case of Posterior Interosseous Nerve Palsy Post-venepuncture. J Coll Physicians Surg Pak 2021; 31(11):1357-1358.

\section{INTRODUCTION}

Wrist drop post-venepuncture is uncommon. There has been reported cases of direct injury to the radial nerve during venepuncture; but to our knowledge, there has never been a case of posterior interosseous nerve (PIN) injury. ${ }^{1,2}$ Our case is unusual as the patient did not develop any pain and presented with PIN injury pattern. Education and training of phlebotomy staff are essential to prevent nerve injury during venepuncture, as it can result in high morbidity despite being a commonly done procedure. We wish to report a case of unusual presentation of a PIN palsy post-routine venepuncture.

\section{CASE REPORT}

A 34-year female, right hand dominant, homemaker, with a newly diagnosed diabetes mellitus, was admitted for diabetic ketoacidosis. She was first seen at the Emergency Department; and there was difficult access with multiple attempts in her blood taking over the antebrachial fossa and forearm. Postvenepuncture, patient did not report any numbness, weakness or tingling sensation of her right forearm or hand at the time of blood taking at the Emergency Department.

Correspondence to: Dr. Raymond D.K. Yeak, Department of Orthopaedic Surgery, Universiti Putra Malaysia,

Serdang, Selangor, Malaysia

E-mail: rayyeak@yahoo.com

Received: November 07, 2019; Revised: April 04, 2020;

Accepted: April 10, 2020

DOI: https://doi.org/10.29271/jcpsp.2021.11.1357
However, upon admission to the ward, she soon noticed that she was unable to fully extend her wrist, fingers and thumb. Sensation over the radial nerve distribution was found to be intact. Haematoma was found over the antecubital fossa, forearm and wrist (Figure 1). Patient was then started on vitamin B complex and cock-up splint was applied. After eight days of no improvement, nerve conduction study was performed. There was slowing of the conduction velocity of the right radial nerve, but the distal motor latency was within normal limits. The electrophysiological study was suggestive of demyelinating right radial neuropathy at the elbow. Patient was diagnosed to have right radial nerve neuropraxia. Upon discharge after 10 days of admission, there was no improvement in wrist, fingers or thumb extension. Six weeks later, patient complained of forearm discomfort, but wrist extension had recovered to normal, whereas, the fingers and thumb extension were still grade 4. During her last follow-up at four months, there was complete recovery of her weakness and radial nerve palsy.

\section{DISCUSSION}

Traumatic venepuncture, due to a direct needle injury, is more common as described in the literature. ${ }^{1}$ Newman et al. found that the incidence of nerve injury during venepuncture was between 1 in $21,000 .{ }^{1}$ A recent study by Tsukuda et al. found that rate of nerve injuries was $0.0015 \%(1 / 67,000)$ for $1,082,053$ venepunctures. $^{2}$ There were eight medial antebrachial cutaneous nerves, five median nerves, two lateral antebrachial cutaneous nerves, and one superficial branch of the radial nerve with no reported case of PIN injury. ${ }^{2}$ Our patient presented with haematoma over the antecubital fossa, forearm and wrist, which led us to suspect a direct needle-induced nerveinjury. 


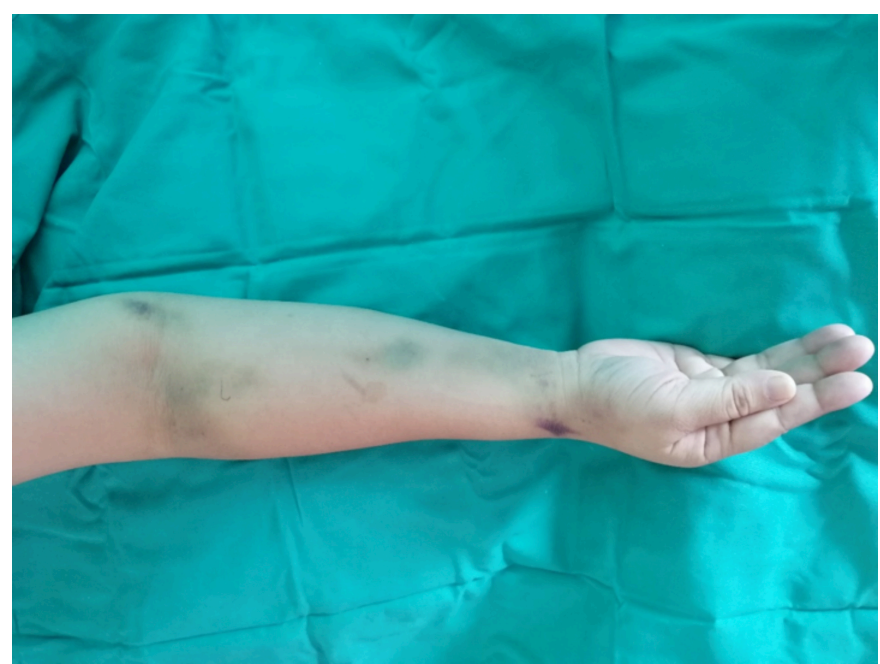

Figure 1: Venepuncture marks and haematoma over the antecubital fossa, forearm and wrist with the fingers kept in a flexed position.

We wish to highlight this case because, despite the transient blood taking session, patient developed neuropraxia, which only resolved after four months. Newman et al. also reported that the majority of the venepuncture-related nerve injury resolved spontaneously. ${ }^{1}$ However, in extreme cases, patients can develop complex regional pain syndrome as reported by Horowitz and other factors besides direct nerve injury should be considered. ${ }^{3,4}$

The radial nerve divides into superficial branch and deep branch at the proximal forearm. The deep branch then pierces the supinator between its two heads to become the PIN. PIN supplies the motor innervation of the posterior forearm muscles. Despite the anatomy of the PIN being at a deeper location than the superficial radial nerve, the patient presented with PIN injury pattern. One possible explanation would be a direct needle-induced nerve injury, which has gone too deep at the antebrachial fossa. The other explanation would be that the damage at the radial nerve could have involved the partial, fascicular branches, which continued distally to form the PIN. ${ }^{5}$

Our case resolved after four months. Despite the favorable outcome, we have since changed our practice. This case has taught us to be more thorough in our consent taking. We have included nerve damage as well as radial nerve damage as a possible complication during venepuncture. We would recommend that a more experienced nurse, doctor or phlebotomist should perform the venepuncture. In addition, besides a full diagnostic workout, the patient should also be referred to a pain specialist, if there were any signs of complex regional pain syndrome post-venepuncture.

Venepuncture is a common procedure, which is done daily at any hospital. Despite it being a common procedure, care should be taken to avoid unintended complications, which in rare instances, can be permanent and disabling. Therefore, the common standard operating procedure should be practiced, as deviations can result in high morbidity to the patient. This case highlights the need for extreme care in taking blood in difficult access cases, as this can lead to significant morbidity to the patient.

\section{PATIENT'S CONSENT:}

The patient has given consent for clinical information to be reported in the journal.

\section{CONFLICT OF INTEREST:}

The authors declared no conflict of interest.

\section{AUTHORS' CONTRIBUTION:}

RDKY: Original idea, prepared the abstract and manuscript writing.

YYY, NMN: Helped prepare the abstract and manuscript writing.

\section{REFERENCES}

1. Newman BH, Waxman DA. Blood donation-related neurologic needle injury: Evaluation of 2 years' worth of data from a large blood center. Transfusion 1996; 36(3): 213-5. doi: 10.1046/j.1537-2995.1996.3639618 2137.x.

2. Tsukuda Y, Funakoshi T, Nasuhara Y, Nagano Y, Shimizu C, Iwasaki N. Venipuncture nerve injuries in the upper extremity from more than 1 million procedures. J Patient Saf 2019; 15(4):299-301. doi: 10.1097/PTS.00000000 00000264.

3. Horowitz SH. Peripheral nerve injury and causalgia secondary to routine venipuncture. Neurology 1994; 44(5):962-4. doi: 10.1212/wnl.44.5.962.

4. Horowitz SH. Venipuncture-induced causalgia: Anatomic relations of upper extremity superficial veins and nerves, and clinical considerations. Transfusion 2000; 40(9): 1036-1040. doi: 10.1046/j.1537-2995. 2000.40091036.x.

5. Bäumer P, Kele H, Xia A, Weiler M, Schwarz D, Bendszus $M$, et al. Posterior interosseous neuropathy: Supinator syndrome vs. fascicular radial neuropathy. Neurol 2016; 87(18):1884-91. doi: 10.1212/WNL.0000000000003287. 